\title{
Levels of Fear in Patients Scheduled for Amputation at The Korle Bu Teaching Hospital, Ghana
}

\author{
Reginald Arthur-Mensah Jnr ${ }^{1}$, Sabina Coffie ${ }^{1}$, Lincoln Tetteh-Ahinakwa ${ }^{2} \&$ Abigail Agartha Kyei ${ }^{1}$ \\ ${ }^{1}$ Department of Nursing and Midwifery, Faculty of Health and Allied Sciences, Pentecost University, Ghana \\ ${ }^{2}$ Department of Mathematics, Kwame Nkrumah University of Science and Technology, Ghana \\ Correspondence: Reginald Arthur-Mensah Jnr, Department of Nursing and Midwifery, Faculty of Health and \\ Allied Sciences, Pentecost University, Ghana.
}

Received: January 21, 2021

Accepted: February 26, 2021

Online Published: March 1, 2021

doi:10.5539/ijps.v13n1p48

URL: https://doi.org/10.5539/ijps.v13n1p48

\begin{abstract}
The purpose of this study was to identify the prevalence and levels of fear in patients scheduled for amputations at the Korle Bu Teaching Hospital (KBTH), Accra, Ghana. A total of 30 patients hospitalized at the surgical wards and the orthopaedic and accident center wards between November 2019 and May 2020 were included in the study. The Surgical Fear Questionnaire (SFQ) was used to measure the levels of fear in the patients the day before surgery.

Findings showed that the major type of amputation was below knee amputation, 16/30 (53.3\%), the main reason for amputation was due to Diabetic foot wounds, $16 / 30(53.3 \%)$, the immediate reaction to the news of the amputation was sadness, 12/30 (40\%). All patients were afraid. Mean (SD) SFQ-s scores were 16.23 (9.22) and mean (SD) SFQ-1 scores were 19.40 (9.65). Though the levels of fear were generally low, long term fear was higher among the patients than the short-term fear consequences of the surgery. Gender was significant associated with patients' immediate reaction to fear. Age was significantly associated with the levels of fear.

We propose multidisciplinary interactions and well-thought-out rehabilitation programs to enhance patient outcomes and improve the quality of life of amputees.
\end{abstract}

Keywords: amputation, Ghana, Korle Bu Teaching Hospital (KBTH), long term fear, short term fear, Surgical Fear Questionnaire (SFQ)

\section{Introduction}

Amputation is the surgical removal of all or part of a limb (Solgajová, Sollár, \& Vörösová, 2015). It is also the condition of disability resulting from the irreversible loss of one or more limbs (Solgajová et al., 2015). Amputation as a surgical option is resorted to in conditions where salvaging a limb is unlikely, and the remaining part of the limb tissue needs excision (Smith \& Skinner, 2014; Uustal, 2015). Surgical amputation of a limb can be an elective procedure or an emergency procedure (Sahu, Sagar, Sarkar, \& Sagar, 2016).

The typical indications of amputation include trauma, diseases and neoplasms (Attinger \& Brown, 2012). Traumatic limb amputation can include motor vehicle accidents, railway track accidents and machinery injury (Tintle et al., 2010). Some Non-Communicable Diseases (NCDs), example diabetic foot wounds represent a condition when the affected foot may require amputation when putrefaction starts to appear (Attinger \& Brown, 2012; McKenzie, Simpson, \& Stewart, 2010). An abnormal new mass of tissue that serves no purpose in any part of the body may also be cut off (Attinger \& Brown, 2012).

\section{Literature Review}

Amputation is a major health burden on the affected individuals, families and the society. For the individual, it may result in physical disabilities, bodily disfigurement and psychosocial impacts (Shahmansouri, Koivula, Ahmadi, Arjmandi, \& Karimi, 2012). Evidence from amputation indicate that traumatic loss of a limb is typically equated with loss of spouse, loss of one's perception of wholeness, symbolic castration and even death (Sahu et al., 2016). This may result in the patient being severely emotionally devastated and result in poor quality of life (Sinha \& Van Den Heuvel, 2011). Depression and anxiety are also common problems among patients who have suffered from amputation (Sahu et al., 2016). The loss of the limb may cause distress not only 
due to the loss of a body part but also due to the role limitation and the need for adjustment to the changed lifestyle options. The individual undergoing amputation may be at risk of developing depressive disorders due to multiple factors such as feelings of loss, self-stigma and difficulty in coping with the impairment. Prevalence of psychiatric disorders among amputees has been found to be in the range of $2 \%-95 \%$ including depression rates of $10.4 \%-63 \%$, posttraumatic stress disorder $3.3 \%-56.3 \%$, and phantom limb phenomenon $14 \%-92 \%$ (Muzaffar, Mansoor, Arifa, \& Margoob, 2012). Further, if amputation was induced by trauma, the distressing events leading to the amputation, may induce symptoms of posttraumatic stress disorder (PTSD) and fear (Abeyasinghe et al., 2012; Durmus et al., 2015; Mckechnie \& John 2014).

Surgical fear is a well recognizable emotional state for many patients waiting for amputation. Apotemnophobia and anxiety are to be expected amongst such patients scheduled for this surgical modality (Shahmansouri et al., 2012; Gallagher \& McKinley, 2007). Surgical fear has been associated with increased postoperative pain, increased use of analgesia, poor postoperative recovery, decreased physical functioning and impaired psychosocial state (McKenzie et al., 2010; Theunissen, Peters, Bruce, Gramke, \& Marcus, 2012; Theunissen et al., 2018; Wilson et al., 2015). Fear can adversely affect surgical patients' existing well-being as well as their Intensive Care Unit and recovery periods (Shahmansouri et al., 2012). Throughout the operative course, not only does fear persist but it also intensifies. These can affect physiological vital signs such as increase in blood pressure, arterial damage, irregular heart rhythms, platelet reactivity, decreased heart variability and increased pro-inflammatory markers (Theunissen et al., 2018).

Notwithstanding, assessment of surgical fear is important because it is the initial and primal step towards preoperative management of patients. It in turn, is likely to increase postoperative personal well-being and function.

In Ghana, there are no known reports in the existing literature on the levels of fear amongst patients scheduled for amputation across health facilities in the country. A better understanding of fear is vitally important for decision-making about the appropriate and timely intervention and provision of management resources aimed at reducing patients' fears. As such, this study is the first of its kind to identify the prevalence of fear and measure its heights in patients scheduled for amputation in Ghana's biggest tertiary health facility, the Korle Bu Teaching Hospital (KBTH).

\section{Methods}

\subsection{Research Design}

The current study adopted a prospective cross-sectional study to assess the prevalence and levels of fear in patients scheduled for amputation at KBTH. The Surgical Fear Questionnaire (SFQ) was used to measure the short-term fear (SFQ-s) and long-term fear (SFQ-1) among patients scheduled for amputation. SFQ-scores were assessed the day before the surgery. Demographic predictors of fear were also assessed.

\subsection{Study Sites}

The surgical wards and the orthopaedic and accident center wards of the surgical department of the KBTH were the study sites.

\subsection{Study Sample}

Patients who had been referred to the surgical wards and the orthopaedic and accident center wards for amputation were included in the study. Patients were included if they were 18 years and above and had been scheduled for elective or emergency amputation.

\subsection{Sample Size and Sampling Procedure}

Census recruitment was employed to include patients into the study. All patients who had been scheduled for amputation while on admission in the surgical wards and the orthopaedic and accident center wards during the period of data collection were included. In all, 30 patients were sampled.

\subsection{Data Collecting Instrument}

The Surgical Fear Questionnaire (SFQ) is an eight-item instrument for the assessment of self-reported surgical fear, suitable for general use among all types of adult surgery patients, covering a range of fear of the short-term consequences of surgery (SFQ-s, item 1-4) and fear of the long-term consequences of surgery (SFQ-1, item 5-8). All items are scored on an eleven-point numeric rating scale ranging from 0 (not at all afraid) to 10 (very afraid). This results in a score of 0-40 for each subscale. Items are: afraid of operation, anesthesia, postoperative pain, side effects, health deterioration, failed operation, incomplete recovery and long duration of rehabilitation. Validity and reliability of the SFQ has been previously established (Theunissen et al., 2014). Demographic data 
was also collected from study sample. The questionnaire completed before the surgery assessed age, sex, marital state, employment state and highest educational level attained among study sample. Type of amputation, reason for amputation and how patients felt on first hearing about their amputation was also assessed. Pre- and post-operative management resource questions were also evaluated from patients and their caregivers.

\subsection{Data Collecting Procedure}

Patient recruitment took place from November 2019 to May 2020. Inclusion criteria were patients aged over 18 years and scheduled for elective or emergency amputation. Patients received questionnaires to complete after exhaustive understanding of the study details through the information sheet and personal orientation and signing the informed consent. Patients who could not self-administer the questionnaires were assisted. All data was field edited immediately to correct any inconsistencies.

\subsection{Ethical Consideration}

Prior to the conduct of this study, approval was obtained from the Research unit of the KBTH, the Nursing Administration, the In-service Department and the Head of Department (HOD) of surgery of the KBTH. Signed informed consent was obtained from all participants.

\subsection{Data Analysis}

Descriptive statistics was used to present the demographic details, type of amputation, reason for amputation, immediate reaction to the news of amputation and the pre- and post-operative management resources among the patients. SFQ subscale SFQ-s, item 1-4 and SFQ-1, item 5-8 was determined on an 11-point numeric rating scale ranging from 0 (not at all afraid) to 10 (very afraid). This resulted in a score of $0-40$ for each subscale. The Chi-square test, the independent $T$ test and ANOVA were used to determine significant associations between the demographic details and the levels of fear among patients. The Paired sample T-test was conducted to determine the significant difference in receiving counselling before the surgery and receiving counselling after the surgery among patients.

\section{Results}

\subsection{Demographic Details of Patients}

Between November 2019 and May 2020 patients scheduled for amputation were sampled for the study. A total of 30 patients were recruited within the period. $21 / 30(70 \%)$ were recruited from the surgical ward and 9/30 (30\%) were recruited from the orthopaedic and accident center wards (see Table 1). They comprised 16/30 $(53.3 \%)$ males and $14 / 30(46.7 \%)$ females. Majority of the patients, $19 / 30(63.3 \%)$ were above 50 years. Patients who had attained up to tertiary level in education also accounted for the majority, 13/30 (43.3\%) who those attaining up to basic level education accounting for the least, $1 / 30$ (3.3\%). About $50 \%$ of patients were entrepreneurs involved in diverse crafts for livelihood, 11/30 (36.7\%) were unemployed and the rest were state and private sector employees (see Table 1).

Table 1. Demographic details of patients

\begin{tabular}{lll}
\hline Demographic details & $\boldsymbol{f}$ & $\boldsymbol{\%}$ \\
\hline Gender & 16 & 53.3 \\
Male & 14 & 46.7 \\
Female & $\mathbf{3 0}$ & $\mathbf{1 0 0}$ \\
Total & & \\
\hline Age (years) & 1 & 3.3 \\
$<20$ & 4 & 13.3 \\
$21-30$ & 4 & 13.3 \\
$31-40$ & 2 & 6.7 \\
$41-50$ & 19 & 63.3 \\
$>51$ & 30 & 100 \\
Total & & \\
\hline Marital status & 13 & 43.3 \\
Single & 13 & 43.3 \\
Married &
\end{tabular}




\begin{tabular}{lll} 
Divorced & 1 & 3.3 \\
Widow/widower & 3 & 10 \\
Total & $\mathbf{3 0}$ & $\mathbf{1 0 0}$ \\
\hline Level of education & & \\
No formal education & 4 & 13.3 \\
Basic education & 1 & 3.3 \\
JHS & 4 & 13.3 \\
SHS & 8 & 26.7 \\
Tertiary education & 13 & 43.3 \\
Total & $\mathbf{3 0}$ & $\mathbf{1 0 0}$ \\
\hline Occupation & & \\
Unemployed & 11 & 36.7 \\
Doing own business & 15 & 50 \\
Government employee & 2 & 6.7 \\
Private sector employee & 2 & 6.7 \\
Total & $\mathbf{3 0}$ & $\mathbf{1 0 0}$ \\
\hline Ward & & 70 \\
Surgical & 21 & 30 \\
Orthopaedic & 9 & $\mathbf{1 0 0}$ \\
Total & $\mathbf{3 0}$ & \\
\hline
\end{tabular}

Note.

f-frequency

$\%$-Percentage

JHS-Junior High School

SHS-Senior High School

\subsection{Type of Amputation among Patients}

The types of amputation among patients are presented in table 2. The main type of amputation recorded was below knee amputations. This was followed by above knee amputations and upper limb amputations.

Table 2. Types of amputation among patients

\begin{tabular}{lll}
\hline Types of amputation & $\boldsymbol{f}$ & $\mathbf{\%}$ \\
\hline Above knee amputation & 11 & 36.7 \\
Below knee amputation & 16 & 53.3 \\
Upper limb amputation (Below elbow disarticulation) & 1 & 3.3 \\
Upper limb amputation (Digits disarticulation) & 1 & 3.3 \\
Upper limb amputation (Shoulder disarticulation) & 1 & 3.3 \\
Total & $\mathbf{3 0}$ & $\mathbf{1 0 0}$ \\
\hline
\end{tabular}

Note.

f-frequency

$\%$-Percentage

\subsection{Reason for Amputation among Patients}

The reason for amputation among patients is presented in table 3 . The major reason recorded among the patients was amputation due to diabetic foot wound which had become gangrenous from putrefaction of non-healing ulcers on the right leg. This was followed by amputation due to diabetic foot wound which had become gangrenous from putrefaction of non-healing ulcers and sepsis on the left leg and amputation due to acute limb ischemia from vasoconstriction of the right leg. 
Table 3. Reason for amputation among patients

\begin{tabular}{lll}
\hline Reason for amputation & $\boldsymbol{f}$ & $\mathbf{\%}$ \\
\hline Due to a right mangled leg & 1 & 3.3 \\
Due to a right necrotic foot & 1 & 3.3 \\
Due to acute limb ischemia of the right leg & 3 & 10 \\
Due to cellulitis ulcer on the right leg & 1 & 3.3 \\
Due to diabetic foot wound on the left leg & 7 & 23.3 \\
Due to diabetic foot wound on the right leg & 9 & 30 \\
Due to fracture of left fingers & 1 & 3.3 \\
Due to fracture of the right leg & 2 & 6.7 \\
Due to gangrene of the left big toe & 1 & 3.3 \\
Due to mangled right hand & 1 & 3.3 \\
Due to polytrauma sepsis of the left leg & 1 & 3.3 \\
Due to sarcoma in the left armpit & 1 & 3.3 \\
Due to unhealed boil on the left leg & 1 & 3.3 \\
Total & $\mathbf{3 0}$ & $\mathbf{1 0 0}$ \\
\hline
\end{tabular}

Note.

f-frequency

$\%$-Percentage

4.4 Patients' Immediate Reaction on Hearing about Their Amputation Procedure

The foremost reaction from patients on hearing they were going to be amputated were feelings of intense sadness. Others were indifferent because they opted for the procedure for their own good whilst the rest became very scared, worried, aggressive, anxious and confused (see Table 4). A chi-square test for significance showed that gender was significantly associated with patients' first reaction on hearing about their amputation $\left(\chi^{2}=6.830\right.$, $P=0.033)$.

Table 4. Patients' immediate reaction on hearing about their amputation

\begin{tabular}{lll}
\hline Patients' immediate reaction on hearing about their amputation & $\boldsymbol{f}$ & $\mathbf{\%}$ \\
\hline I became very scared, worried, aggressive, anxious, confused & 8 & 26.7 \\
I felt indifferent because i opted for it for my own good & 10 & 33.3 \\
I felt very sad & 12 & 40 \\
Total & $\mathbf{3 0}$ & $\mathbf{1 0 0}$ \\
\hline
\end{tabular}

Note.

f-frequency

$\%$-Percentage

\subsection{Prevalence and Levels of Fear among Patients}

The SFQ was used to assess the prevalence and levels of fear among the patients. Aggregate scores for short term fear (SFQ-s) ranged from a minimum of 2 to a maximum of 36. Aggregate scores for long term fear (SFQ-1) ranged from a minimum of 5 to a maximum of 40 . This indicated the prevalence of considerable levels of fear with one patient being completely afraid of the long-term consequences of the surgery. The difference of the range between the SFQ-s and SFQ-1 also showed a higher difference for SFQ-1. Mean (SD) SFQ-s scores were 16.23 (9.22) and mean (SD) SFQ-1 scores were 19.40 (9.65). This indicated both levels of fear were low. However, long term fear was higher among the patients than the short-term consequences of the surgery.

An independent T-test and ANOVA test was performed to determine significant differences in the SFQ-S and SFQ-1 among gender. Mean scores for males and females were 18.69 and 13.43 respectively for SFQ-S and 20.75 and 17.86 respectively for SFQ-1. Results for the t-test shows that there was no significant difference between 
gender for SFQ-s (mean difference $=5.259, P=0.451)$ and SFQ-1 (mean difference $=2.893, P=0.131$ ).

ANOVA test for significant difference in the levels of fear among age, marital status, level of education, occupation and type of amputation showed significant difference with only age for the SFQ-s ( $\mathrm{F}=3.516$, $P=0.032$ ). However, a post-hoc test to determine where the differences lie could not be performed due to fewer cases in the age categories.

\subsection{Pre-and/or Post-Operative Management Resources}

We further assessed whether any form of pre- and/or post-operative management of surgical fear was done for the patients and/or their caretakers. Table 5 presents the results. A paired sample T-test showed that there was no significant difference in receiving counselling before the surgery and after the surgery among patients $(P=0.476)$.

Table 5. Pre- and/or post-operative management resource

\begin{tabular}{llll}
\hline Pre- and post-operative management resource & & $\boldsymbol{f}$ & $\mathbf{\%}$ \\
\hline Did you receive any counselling before the surgery? & Yes & $\mathbf{2 7}$ & $\mathbf{9 0}$ \\
& No & 3 & 10 \\
Total & & 30 & 100 \\
\hline Did you receive any counselling after the surgery? & Yes & $\mathbf{2 5}$ & $\mathbf{8 3 . 3}$ \\
& No & 5 & 16.7 \\
Total & & 30 & 100 \\
\hline Did your caretakers receive any counselling on how to support you before the surgery? & Yes & $\mathbf{2 6}$ & $\mathbf{8 6 . 7}$ \\
& No & 4 & 13.3 \\
Total & & 30 & 100 \\
\hline Did your caretakers receive any counselling on how to support you after the surgery? & Yes & $\mathbf{2 5}$ & $\mathbf{8 3 . 3}$ \\
& No & 5 & 16.6 \\
Total & & 30 & 100 \\
\hline Were you educated on how to prevent complications after the surgery? & Yes & $\mathbf{2 5}$ & $\mathbf{8 3 . 3}$ \\
& No & 5 & 16.7 \\
Total & & 30 & 100 \\
\hline Were your caretakers educated on how to prevent complications to you after the & Yes & $\mathbf{2 9}$ & $\mathbf{9 6 . 7}$ \\
surgery? & No & 1 & 3.3 \\
Total & & 30 & 100 \\
\hline
\end{tabular}

Note.

f-frequency

$\%$-Percentage

\section{Discussion}

The meaning of amputation reflects the diversity of patients and their experiences. For example, a previously bed bound person who receives an amputation is likely to experience amputation differently from an elite athlete who requires an amputation. The ability to cope with an amputation will be affected by patients' levels of fear, pain, level of disability, adequacy of cosmesis, cultural issues, presence of social support, the reactions of caregivers and other loved ones (Bhuvaneswar, Epstein, \& Stern, 2007; Wilson et al., 2015). This study sought to evaluate the prevalence and levels of fear in patients scheduled for amputation at the KBTH, Accra, Ghana. As secondary aims, we examined the types of amputation, the reason for amputation, patients' immediate reaction to the news of amputation and the management resources in place aimed at making the entire process of the surgery a more tolerable one.

In light of the on-going pandemic, our sample size was affected due to lockdown restrictions. Data collection was temporarily halted during the lockdown and ensued after restrictions were lifted. However, after the period, access to patients became limited due to strict preventive measures of the pandemic at the KBTH. As well, the safety of the researchers was also imperative and thus the collected sample of 30 patients were used. 
The major type of amputation recorded in this study was below knee amputations with Diabetes being the major reason for the amputation. According to the global disability burden estimates by Zhang et al., (2020), male-to-female years lived with disability, (YLD) ratios ranged from 0.96 for neuropathy to 1.93 for foot ulcers. The 50-69-year age-group accounted for $47.8 \%$ of all YLDs from Diabetes related lower extremity complications (DRLEC). The International Diabetes Federation (IDF) estimated that 1 in every 11 adults (451 million) had diabetes in 2017, and 1 in 10 (693 million) will have diabetes by 2045 (Cho et al., 2018). People with diabetes are at high risk of developing a range of complications, including cardiovascular, kidney, eye, and lower-extremity complications (Harding, Pavkov, Magliano, Shaw, \& Gregg, 2019; Gregg, Sattar, \& Ali, 2016). Arguably, the most disabling of these are the lower-extremity complications of peripheral neuropathy, foot ulceration and amputation (Armstrong, Boulton, \& Bus, 2017). Diabetic foot wounds are one of the most common, costly and severe complications of diabetes. According to the IDF, amputation in people with diabetes is 10 to 20 times more common than in people without diabetes. It is estimated that in every 30 seconds a lower limb or part of a lower limb is lost somewhere in the world and $85 \%$ of these are as a consequence of diabetes (Geiss et al., 2018; IDF, 2017). Moreover, of persons with diabetes who have had a leg amputation, up to 55\% will require amputation of the second leg within 2 to 3 years (Zhang et al., 2020).

Further, it is estimated that below-knee amputations are the most common amputations, representing $71 \%$ of all dysvascular amputations. There was also a 47\% expected increase in below knee amputations from 1995 to 2020 (Zhang et al., 2020). Unfortunately, nearly half of the people who have amputation due to vascular disease will die within the next 5 years (Bhuvaneswar et al., 2007; Harding et al., 2019). This is higher than the five-year mortality rates for breast cancer, colon cancer and prostate cancer.

Immediate reactions to the news of amputation vary. These depend on whether the amputation was by consent and/or planned, occurred within the context of a chronic medical illness or sudden onset of infectious diseases and injuries due to accidents (Bhuvaneswar et al., 2007). The context of amputation also has an impact on the psychological sequelae of the patient. Classic among this, are the characteristic stages of grief (Bhuvaneswar et al., 2007; Parkes, 1975). They include denial, anger, bargaining, depression and acceptance (Kubler-Ross, 1969). For patients whose amputation come in the wake of a sudden accidental trauma, there may be little or no time to pass through these stages. Such patients experience only a few hours of semi consciousness, the fearful awareness before anesthesia, the pain of the surgery and awakening as an amputee. The feelings and/or emotions of intense sadness was the main immediate reaction recorded in our sample. This is reasonable among the sample and is reflective of the obvious lifestyle changes the amputation will bring.

The prevalence and levels of fear was determined using the SFQ. Fear is the human emotion that is triggered by a perceived threat (Ropeik, 2004). It is a physiological response experienced in anticipation of some specific pain or danger (Southwick \& Charney, 2012). Short-term fear is the present habituation and expression of that physiological response while long term fear is the orientation of that physiological response to be expressed in a later time (Ropeik, 2004). Fear is common with amputation (Shahmansouri et al., 2012; Gallagher \& McKinley, 2007). Fear, before and following amputation results from the reaction to the surgical procedure and to the sudden disability subsequently (Bhuvaneswar et al., 2007). Our results revealed low SFQ-s and SFQ-1 fear scores. However, long term fear was higher among the patients than the short-term consequences of the surgery. Impliedly, most of the patients were in the present time not fearful of the procedure they were going to have. Fear of the surgery remained stable. The generally low levels of surgical fear may be due to the refined preoperative procedure of managing patients' fears about amputation at the surgical department of the KBTH as evidenced by our findings. Almost all patients and their caregivers received counselling before the surgery and after the surgery and both were educated on how to care and prevent complications to the amputated limb. At the surgical department, prior to the procedure, patients and their caregivers were referred to a clinical psychologist from the Psychiatry department of the KBTH for counselling sessions before the procedure was done. For elective procedures, the surgery was delayed until satisfactory compliance from the patients and their caregivers was obtained. After the procedure, patients and their caregivers were again referred to a clinical psychologist for counselling on positive coping lifestyles. We concur this could have had a strong positive outlook in the preparation and outcome of the holistic amputation experience. However, a strong multidisciplinary team approach of other specialists in the optimal management of amputation was lacking.

Multidisciplinary management resources before and after amputation involves planning a therapy regimen, managing the immediate postoperative period, planning for an appropriate level of care after discharge and educating the patient and caregivers on the care and prevention of complications. Be that as it may, good mobility function, successful coping strategies and enhanced quality of life are possible and should be presented to amputees as realistic goals following amputations. 


\section{Contribution of Study}

This study is the first to report on the prevalence and levels of fear in patients scheduled for elective and/or emergency amputations in Ghana. It has thus provided preliminary results on the prevalence and levels of fear in patients scheduled for amputation in Ghana. The study establishes below knee amputation and diabetes as the common type and reason for amputation globally. The strength of our findings further reveal evidence for the validity of the subscales of the SFQ. The SFQ is sensitive to detect differences in fear at all time points.

\section{Limitations and Areas for Further Study}

We did not measure the time course of the prevalence and levels of fear in our sample. Fear was measured the day before surgery. Measuring different time course of fear before or after the surgery could have provided information on the variations and intensity of fear within the period. We hypothesized fear to be heightened close to the time of surgery. Also, the restraint in our sample size hindered the ability to assess potential associations between the demographic characteristics and levels of fear and other markers of amputation from large data. We suggest further studies into the psychological and psychosocial factors and surgical outcomes in patients scheduled for amputation and the adaptive or coping lifestyle of amputees in Ghana.

\section{Conclusions}

Below-knee amputations continue to be the most performed type of amputation. Diabetes additionally remains the leading cause of amputation. Our findings contribute to the data that Diabetes-Related Lower-Extremity Complications (DRLEC) are a large and growing contributor to the amputation related disability burden worldwide and disproportionately affect males and middle- to older-aged populations. These findings should facilitate policymakers worldwide to target strategies at improving livelihoods through better nutrition towards preventing the burden of Non-Communicable Diseases (NCDs) especially Diabetes. Additionally, the Psychiatry department of the KBTH deserves commendation.

\section{Implication for Practice}

Both the antecedent circumstances preceding the amputation and the irreversible procedure of amputation itself may contribute to the occurrence of physical and psychological distress. There is the need for the implementation of a holistic multidisciplinary team approach in the management of amputation for a comprehensive evaluation of the patient before and after the surgery. This requires timely medical interactions and well-thought-out rehabilitation programs to enhance patient outcomes and quality of life.

\section{Recommendations}

Individuals should adapt their diets to favourable options that will reduce the plight of the development of NCDs. Fear in amputation is controllable and thus there should be the focus on building up patient resilience, building up caregiver resilience, supportive treatment, treatment of preexisting psychiatric disorders, involvement in rehabilitation programs and reinforcement of the positive coping styles. Employment programs for amputees may also be an important post-operative management resource.

\section{Conflict of Interest}

None.

\section{Acknowledgements}

We thank the nursing staff of the surgical wards and the orthopaedic and accident center wards of the surgical department of the KBTH. We are also grateful to the patients for taking part in this study.

\section{References}

Abeyasinghe, N. L., de Zoysa, P., Bandara, K. M., Bartholameuz, N. A., \& Bandara, J. M. (2012). The prevalence of symptoms of post-traumatic stress disorder among soldiers with amputation of a limb or spinal injury: A report from a rehabilitation centre in Sri Lanka. Psychol Health Med., 17, 376-381. https://doi.org/10.1080/13548506.2011.608805

Armstrong, D. G., Boulton, A. J. M., \& Bus, S. A. (2017). Diabetic foot ulcers and their recurrence. N. Engl. J. Med., 376, 2367-2375. https://doi.org/10.1056/NEJMra1615439

Attinger, C. E, \& Brown, B. J. (2012). Amputation and ambulation in diabetic patients: Function is the goal. Diabetes Metab Res Rev., 28(Suppl 1), 93-96. https://doi.org/10.1002/dmrr.2236

Bhuvaneswar, C. G., Epstein, L. A., \& Stern, T. A. (2007). Reactions to Amputation: Recognition and Treatment. Prim Care Companion. J Clin Psychiatry, 9(4), 303-309. https://doi.org/10.4088/PCC.v09n0408 
Cho, N. H., Shaw, J. E., Karuranga, S. et al. (2018). IDF Diabetes Atlas: global estimates of diabetes prevalence for 2017 and projections for 2045. Diabetes Res Clin Pract, 138, 271-281. https://doi.org/10.1016/j.diabres.2018.02.023

Durmus, D., Safaz, I., Adigüzel, E., Uran, A., Sarisoy, G., Goktepe, A. S. et al. (2015). The relationship between prosthesis use, phantom pain and psychiatric symptoms in male traumatic limb amputees. Compr Psychiatry, 59, 45-53. https://doi.org/10.1016/j.comppsych.2014.10.018

Gallagher, R., \& McKinley, S. (2007). Stressors. Am J., $16(3), \quad$ 248-257. https://doi.org/10.4037/ajcc2007.16.3.248

Geiss, L. S., Li, Y., Hora, I., Albright, A., Rolka, D., \& Gregg, E. W. (2018). Resurgence of diabetes-related nontraumatic lower extremity amputation in the young and middle-aged adult US population. Diabetes Care. https://doi.org/10.2337/dc18-1380

Gregg, E. W., Sattar, N., \& Ali, M. K. (2016). The changing face of diabetes complications. Lancet Diabetes Endocrinol, 4, 537-547. https://doi.org/10.1016/S2213-8587(16)30010-9

Harding, J. L., Pavkov, M. E., Magliano, D. J., Shaw, J. E., \& Gregg, E. W. (2019). Global trends in diabetes complications: a review of current evidence. Diabetologia, 62, 3-16. https://doi.org/10.1007/s00125-018-4711-2

International Diabetes federation. (2017). IDF Diabetes Atlas. 8th edition. Brussels.

Kubler-Ross, E. (1969). On death and dying. New York, NY: Simon \& Schuster.

Mckechnie, P. S., \& John, A. (2014). Anxiety and depression following traumatic limb amputation: A systematic review. Injury., 45, 1859-1866. https://doi.org/10.1016/j.injury.2014.09.015

McKenzie, L. H., Simpson, J., \& Stewart, M. (2010). A systematic review of pre-operative predictors of postoperative depression and anxiety in individuals who have undergone coronary artery bypass graft surgery. Psychol Health Med, 15, 74-93. https://doi.org/10.1080/13548500903483486

Muzaffar, N., Mansoor, I., Arifa, H., \& Margoob, M. A. (2012). Psychiatric comorbidity in amputees with average sociodemographic status and the role of theologic and family support in a conflict zone. Australas $J$. Disaster Trauma Stud., 2012, 31-38.

Parkes, C. M. (1975). Psycho-social transitions: comparison between reactions to loss of limb to loss of spouse. Br. J. Psychiatry, 127, 204-210. https://doi.org/10.1192/bjp.127.3.204

Ropeik, D. (2004). The consequences of fear. EMBO Reports, 5(1), 56. https://doi.org/10.1038/sj.embor.7400228

Sahu, A., Sagar, R., Sarkar, S., \& Sagar, S. (2016). Psychological effects of amputation: A review of studies from India. Industrial psychiatry journal, 25(1), 4-10. https://doi.org/10.4103/0972-6748.196041

Shahmansouri, N., Koivula, M., Ahmadi, S. H., Arjmandi, A., \& Karimi, A. (2012). Fear, Anxiety, and Beliefs about Surgery in Candidates Patients for Coronary Artery Bypass Grafting. Euro. J. Exp. Bio., 2(5), 1750-1754.

Sinha, R., \& Van Den Heuvel, W. J. (2011). A systematic literature review of quality of life in lower limb amputees. Disabil Rehabil., 33, 883-899. https://doi.org/10.3109/09638288.2010.514646

Smith, D. G., \& Skinner, H. B. (2014). Amputations. Current Diagnosis and Treatment in Orthopedics. 5th ed. 11. In Skinner, H. B., \& McMahon, P. J. (Eds.). New York: The McGraw-Hill Companies.

Solgajová, A., Sollár, T., \& Vörösová, G. (2015). Gender, age and proactive coping as predictors of coping in patients with limb amputation. Kontakt., 17, e67-72. https://doi.org/10.1016/j.kontakt.2015.01.005

Southwick, S., \& Charney, D. (2012). Resilience. The science of mastering life's greatest challenges. New York: Cambridge University Press. https://doi.org/10.1017/CBO9781139013857

Theunissen, M., Jonker, S., Schepers, J., Nicolson, N. A., Nuijts, R., Gramke, H-F. et al. (2018). Validity and time course of surgical fear as measured with the Surgical Fear Questionnaire in patients undergoing cataract surgery. PLoS ONE, 13(8), e0201511. https://doi.org/10.1371/journal.pone.0201511

Theunissen, M., Peters, M. L., Bruce, J., Gramke, H. F., \& Marcus, M. A. (2012). Preoperative anxiety and catastrophizing: a systematic review and meta-analysis of the association with chronic postsurgical pain. Clin J. Pain, 28, 819-841. https://doi.org/10.1097/AJP.0b013e31824549d6 
Theunissen, M., Peters, M. L., Schouten, E. G., W., Fiddelers, A. A. A., Willemsen, M. G. A. et al. (2014). Validation of the Surgical Fear Questionnaire in Adult Patients Waiting for Elective Surgery. PLOS ONE, 9(6), e100225. https://doi.org/10.1371/journal.pone.0100225

Tintle, S. M., Keeling, J. J., Shawen, S. B., Forsberg, J. A., \& Potter, B. K. (2010). Traumatic and trauma-related amputations: Part I: General principles and lower-extremity amputations. J Bone Joint Surg Am., 92, 2852-2868. https://doi.org/10.2106/JBJS.J.00257

Uustal, H. (2015). Lower limb amputation, rehabilitation, and prosthetic restoration. Current Diagnosis and Treatment: Physical Medicine and Rehabilitation. In Maitin, I. B., \& Cruz, E. (Eds.). New York: McGraw-Hill Education.

Wilson, C. J., Mitchelson, A. J., Tzeng, T. H., El-Othmani, M. M., Saleh, J., Vasdev, S. et al. (2015). Caring for the surgically anxious patient: a review of the interventions and a guide to optimizing surgical outcomes. Am J. Surg. https://doi.org/10.1016/j.amjsurg.2015.03.023

Zhang, Y., Lazzarini, P. A., McPhail, S. M., van Netten, J. J., Armstrong, D. G., \& Pacella, R. E. (2020). Global Disability Burdens of Diabetes-Related Lower-Extremity Complications in 1990 and 2016. Diabetes Care. https://doi.org/10.2337/dc19-1614

\section{Copyrights}

Copyright for this article is retained by the author(s), with first publication rights granted to the journal.

This is an open-access article distributed under the terms and conditions of the Creative Commons Attribution license (http://creativecommons.org/licenses/by/4.0/). 